\title{
3. Öffentliche Entwicklungszusammenarbeit
}

\section{Hilmar Stetter}

\section{OpenEdition}

\section{Journals}

Electronic version

URL: http://journals.openedition.org/sjep/981

DOI: 10.4000/sjep.981

ISSN: 1663-9677

\section{Publisher}

Institut de hautes études internationales et du développement

\section{Printed version}

Date of publication: 31 août 1982

Number of pages: 81-102

ISSN: $1660-5926$

\section{Electronic reference}

Hilmar Stetter, « 3. Öffentliche Entwicklungszusammenarbeit », Schweizerisches Jahrbuch für Entwicklungspolitik [Online], 2 | 1982, Online erschienen am: 28 Januar 2013, abgerufen am 08 September 2020. URL : http://journals.openedition.org/sjep/981 ; DOI : https://doi.org/10.4000/sjep. 981 


$$
\begin{aligned}
& \text { 1. Tahresübersicht }
\end{aligned}
$$

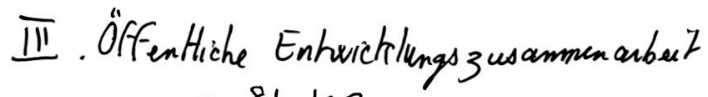

$$
\begin{aligned}
& \text { p. 81.102. }
\end{aligned}
$$

\section{OEFFENTLICHE ENTWICKLUNGSZUSAMMENARBEIT}

Die öffentliche Entwicklungszusammenarbeit der Schweiz war 1981 von den linearen Kürzungen des Bundesbudgets um 10\% betroffen. Für 1982 wurde vom Parlament zudem eine weitere Kürzung um $8 \%$ beschlossen. Sie stagniert damit - bei nominal leicht wachsendem Volumen im Verhältnis zum BSP und bleibt anteilsmässig international unterdurchschnittlich. Im Dezember 1981 genehmigte das Parlament einen neuen Rahmenkredit von 360 Mio Fr. für Massnahmen der internationalen humanitären Hilfe. Schliesslich stimmte der Bundesrat dem Vertrag zwischen dem Bund und der "Intercooperation" zu.

\section{DIE AUFWENDUNGEN FUER DIE OEFFENTLICHE ENTWICKLUNGSZUSAMMENARBEIT 1981}

1981 stellte die Schweiz für ihre öffentliche Entwicklungshilfe (Bund, Kantone und Gemeinden zusammengefasst) 452,6 Mio Fr. zur Verfügung. Das sind $0,24 \%$ des BSP $-0,01 \%$ mehr als 1980 . Für 1982 beläuft sich das Budget auf 481,2 Mio Fr., was einer Erhöhung um 5,8\% gleichkommt. Der Anteil am BSP dürfte sich auf $0,24 \%$ belaufen (1).

Mit ihren - gemessen am BSP - stagnierenden Ausgaben für die Entwicklungszusammenarbeit liegt die Schweiz unter dem Durchschnitt aller Industrieländer. Sie klassierte sich $1981 \mathrm{im} \mathrm{15}$. Rang aller DAC-Länder. Auch pro Kopf der Bevölkerung berechnet leistet die Schweiz wenig öffentliche Entwicklungshilfe : $71 \mathrm{Fr}$. Sie ist weit davon entfernt, das Ziel der UN-Entwicklungsstrategie für die 80 er Jahre zu erreichen, nähmlich $0,7 \%$ des BSP für die Entwicklungszusammenarbeit aufzuwenden. Dieser Strategie hat sich die Schweiz im übrigen angeschlossen. Und der Beitrag der Schweiz an die gesamte öffentliche Entwicklungshilfe der DAC-Länder belief sich 1981 auf $0,93 \%$.

Die gesamte Entwicklungszusammenarbeit des Bundes wird durch verschiedene Rahmenkredite gewährleistet, welche von verschiedenen Departementen 
verwaltet werden. Es handelt sich um :

- den Rahmenkredit von 1,650 Mio Schweizer Franken vom 8. Dezember 1980 für technische Zusammenarbeit und Finanzhilfe;

- den Rahmenkredit von 270 Mio Schweizer Franken vom 14. März 1979 für die internationale humanitäre Hilfe. Dieser Kredit ist am 31. März 1982 abgelaufen. Ein neuer Kredit von 360 Mio Schweizer Franken wurde am 3. Dezember 1981 bewilligt und ist seit April 1982 in Kraft;

- den Rahmenkredit von 300 Mio Schweizer Franken vom 26. September 1979 für Kapitalerhöhungen der Interamerikanischen, der Afrikanischen und der Asiatischen Entwicklungsbank;

- den Rahmenkredit von 200 Mio Schweizer Franken vom 28. November 1978 über die Finanzierung von wirtschafts- und handelspolitischer Entwicklungszusammenarbeit. Er war Mitte Jahr voll verpflichtet, ein neuer Kredit von 350 Mio Fr. wurde dem Parlament vorgelegt;

- den Rahmenkredit von 9,2 Mio Schweizer Franken vom 19. Dezember 1980 für die Gewährung von Stipendien an ausländische Studenten in der Schweiz. Der überwiegende Teil dieses Kredites wird für Studierende aus Ländern der Dritten Welt verwendet (2).

In den Grundzügen seiner Regierungspolitik für die Legislaturperiode 197983 setzte sich der Bundesrat eine substantielle Erhöhung seiner Aufwendungen für die Entwicklungszusammenarbeit zum Ziel. Er wollte eine Annäherung an die durchschnittlichen Aufwendungen aller DAC-Länder - diese belaufen sich auf ca. 0,35\% des BSP - erzielen. Und in den späteren Jahren will er diesen Durchschnitt erreichen. In Zahlen ausgedrückt sind im Finanzplan der laufenden Legislaturperiode die folgenden Gesamtaufwendungen für die öffentliche Entwicklungszusammenarbeit vorgesehen :

\begin{tabular}{lcc} 
Jahr & Höhe (Mio Fr.) & Anteil am BSP \\
\hline 1981 & 487,8 & $0,27 \%$ \\
1982 & 598,8 & $0,31 \%$ \\
1983 & 618,8 & $0,31 \%$
\end{tabular}

Aufgrund der Sparmassnahmen des Bundes von 1980 werden die geplanten Mittel des Bundes für die Entwicklungshilfe jährlich um $10 \%$ reduziert. So beliefen sich die Aufwendungen des Bundes allein (ohne Kantone und Gemeinden) noch auf 446,5 Mio Fr., d.h. 2,6\% des gesamten Budgets oder $0,24 \%$ des BSP. Die Erhöhung gegenüber dem Vorjahr 1980 betrug nominal $10 \%$. Dies ist vor allem auf die Gewährung eines Wirtschaftshilfekredits an die Türkei zurückführen (3). 


\section{Die Aufteilung nach verschiedenen Anteilen}

Der Anteil der technischen Zusammenarbeit an den gesamten Aufwendungen blieb 1981 fast konstant (Erhöhung um 2\%), ebenso wie die Nahrungs- und die humanitäre Hilfe (Senkung um je $2 \%$ ). Einen grösseren Anteil - auf Kosten der Finanzhilfe - hatten die wirtschafts- und handelspolitischen Massnahmen inne (14\% gegenüber $4 \%$ ), während die Finanzhilfe noch $21 \%$ ausmachte (gegenüber $30 \%$ im Vorjahr) (4).

Der Anteil der Geschenke an den gesamten Hilfeleistungen belief sich auf $88,8 \%$, was gegenüber $1980(97,1 \%)$ eine Verminderung bedeutet (5).

Der Anteil der bilateralen Hilfe veränderte sich kaum $164,5 \%$ gegenüber $64,8 \%$ im Vorjahr). Hingegen veränderte sich ihre geographische Verteilung. Wiederum leicht steigern konnte sich der Anteil Afrikas - auf 42,4\%-, während anteilsmässig Lateinamerika leicht und Asien und Ozeanien beachtlich - von 36,6 auf 26,9\% - an Bedeutung verloren. Aussergewöhnlich ist der gestiegene Anteil Europas - von 0,2\% auf 7,5\%. Dies ist wiederum auf die Gewährung eines Wirtschaftshilfekredits an die Türkei zurückzuführen (6).

Teilt man die bilaterale Hilfe nach der Einkommenshöhe der Empfängerländer auf, so haben 1981 die ärmsten Entwicklungsländer weiterhin den grössten Teil der Hilfe - nämlich 39\% - erhalten. Hingegen verschoben sich die weiteren Anteile zugunsten der Entwicklungsländer mit mittlerem Einkommen (22\% gegenüber $16 \% 1980)$. Dies ist vor allem auf die Hilfe an die Türkei zurückzuführen. Die anderen einkommensschwachen Länder erhielten 18\% der bilateralen Hilfe (gegenüber $25 \% 1980$ ) (7).

\section{Die wichtigsten entwicklungspolitischen Entscheide}

Die quantitative Charakteristik besagt nicht alles über die Aktivitäten der schweizerischen Entwicklungszusammenarbeit und die entwicklungspolitischen Entscheide in den vergangenen zwölf Monaten. Denn die zu Beginn des Jahres 1981 ausgelöste Diskussion um die schweizerische Entwicklungszusammenarbeit wurde in den Kommissionen, im Parlament und in den Medien weitergeführt. Dabei wurden weniger die Prinzipien und die Notwendigkeit schweizerischer Entwicklungszusammenarbeit diskutiert, als einzelne Instrumente und Massnahmen. Im einzelnen standen ihre Zusammenarbeit mit der Privatwirtschaft, die Transparenz und die Information der mit der Entwicklungszusammenarbeit beauftragen Bundesstellen im Zentrum der Diskussionen.

Was die Höhe der Entwicklungshilfe anbelangt, so stimmten beide Kammern neben der linearen 10\%-igen Kürzung des Budgets einer weiteren $8 \%$-igen Kürzung zu. Der Antrag Bauer (L/GE) auf vollständige Ausnahme der Entwicklungszusammenarbeit von dieser Kürzung wurde im Ständerat mit 21 zu 8 Stimmen abgelehnt. Im Nationalrat wurde der Antrag Ott (S/BL) auf Ausnahme der technischen Zusammenarbeit von der $8 \%$-igen Kürzung mit 76 zu 59 Stimmen abgelehnt. Auch die Finanzkommission und der Bundesrat 
wandten sich gegen diesen Antrag. Gegen diese Kürzungen beim Budget für die Entwicklungszusammenarbeit wandte sich hingegen die Arbeitsgemeinschaft Swissaid/Fastenopfer/Brot für Brüder/Helvetas. Sie gingen "auf Kosten der Armen" (8).

Schliesslich hatte das Parlament über zwei neue Rahmenkredite zu befinden :

- Rahmenkredit über 350 Mio Fr. zur Weiterführung von wirtschafts- und handelspolitischen Massnahmen im Rahmen der Entwicklungszusammenarbeit. Dieser ersetzt den auslaufenden Kredit von 200 Mio Fr. und soll Massnahmen in den Bereichen Rohstoffe, Handel, Industrialisierung und Finanzen (Mischkredite und Zahlungsbilanzhilfe) für mindestens drei Jahre ermöglichen. Die entsprechenden Auszahlungen werden sich auf die nächsten fünf bis sieben Jahre erstrecken. Der Kredit wurde vom Nationalrat am 23. Juni 1982 befürwortet. Er liegt dem Ständerat in der Herbstsession vor.

- Rahmenkredit über 360 Mio Fr. für internationale humanitäre Hilfe. Er finanziert ebenfalls Massnahmen für mindestens die nächsten drei Jahre und zwar ab 1. April 1982. Der letzte Kredit über 270 Mio Fr. lief an diesem Datum aus.

Im Dezember 1981 stimmte der Bundesrat einem Vertrag zu, welcher die Zusammenarbeit zwischen dem Bund und der neuen Organisation "Intercooperation", deren Träger sieben schweizerische Hilfswerke sind, regelt. "Diese Zusammenarbeit gehört zu jenen Massnahmen, die es dem Bund erlauben sollen, auch bei einem seit einigen Jahren wachsenden Aufgabenvolumen und einem wenig veränderten Personalbestand die Qualität der schweizerischen bilateralen Entwicklungszusammenarbeit aufrecht zu erhalten" (9).

Der Abschluss des Vertrags war eine intensive Diskussion im Parlament und in der Presse vorausgegangen. Ingenieurfirmen und weitere Wirtschaftsvertreter bemängelten bei dieser Gelegenheit die ihrer Meinung nach mangelnden Kontakte der DEH mit der Wirtschaft. Die Verwaltung ihrerseits konnte die Vielfalt der bereits bestehenden Beziehungen aufzeigen und veröffentlichte erstmals Zahlen über die Rückflüsse aus der Entwicklungszusammenarbeit an die Privatwirtschaft. Um den Informationsfluss zur Privatwirtschaft zu verbessern, organisierte die DEH nun eine Früh-Information über Projekte und Programme der Technischen Zusammenarbeit und Finanzhilfe (10).

Die beratende Kommission für die internationale Entwicklungszusammenarbeit verfolgte ebenfalls intensiv die schweizerische Entwicklungspolitik. Besondere Diskussionspunkte waren - zusammen mit der beratenden Kommission für die Handelspolitik - die Erneuerung des Rahmenkredits für wirtschafts- und handelspolitische Massnahmen, der Entscheidprozess für Projekte der technischen Zusammenarbeit und der Finanzhilfe und für die Gewährung von Mischkrediten. In der letzten Sitzung des ersten Halbjahres 1982 beriet die Kommission das Budget 1983 der Entwicklungszusammenarbeit und das Vorgehen bei Projektevaluationen (11). 
Am 17. September 1981 konnte die DEH an ihrer Jahreskonferenz auf eine 20-jährige Tätigkeit zurückblicken. Sie nahm dies zum Anlass, durch einen internationalen Vergleich mit fünf hochindustrialisierten Ländern (Dänemark, Italien, Kanada, Niederlande, Schweden) eine Standortbestimmung vorzunehmen. Auch antwortete sie auf die Kritik an ihrer Tätigkeit. Wie Bundesrat Aubert ausführte, sei die Solidarität mit den Armen und die Hilfe an die Entwicklungsländer grundsätzlich nötig und nützlich. Begleitet war die Jahreskonferenz von der Herausgabe spezieller Publikationen (12).

Schliesslich gab es in der Leitung der mit der Durchführung der Entwicklungszusammenarbeit betrauten DEH eine wichtige personelle Aenderung. Der bisherige Direktor, Botschafter Marcel Heimo, tritt Ende 1982 in den Ruhestand. Der Bundesrat ernannte im Juni 1982 Fritz Staehelin zu seinem Nachfolger. Botschafter Staehelin war seit 1980 Schweizer Botschafter in Tokio, von 1970-75 hatte er die Leitung des Entwicklungsdienstes des heutigen BAWI innegehabt (13).

\section{Die öffentliche Aufwendung im Vergleich} $z u$ den privaten Finanzströmen

Im Gegensatz zu den unterdurchschnittlichen Leistungen an Entwicklungshilfe spielt die Schweiz eine bedeutende Rolle bei den privaten Finanzströmen nach Entwicklungsländern. Sie gehört, in absoluten Zahlen, neben Belgien, Frankreich, der BRD, Italien, Japan, Grossbritannien und den USA zu den wichtigeren Geldgebern für die Entwicklungsländer. Im Vergleich mit dem BSP pro Kopf liegt die Schweiz bei den privaten Kapitalflüssen nach Entwicklungsländern seit mehreren Jahren mit Abstand an der Spitze der DAC-Länder. Der schweizerische Beitrag macht $4 \%$ aller privaten Finanzflüsse der DAC-Länder nach den Entwicklungsländern aus.

1981 waren die Nettozuflüsse von schweizerischem Privatkapital nach Entwicklungsländern etwa neunmal so hoch wie die Entwicklungshilfegelder, nämlich 3.888,5 Mio Fr. Damit beliefen sie. sich auf 2,03\% des BSP von 1981. Der Durchschnitt aller DAC-Länder für die privaten Finanzflüsse lag 1981 bei $0,74 \%$ des BSP (14). Und durchschnittlich betrug der Anteil der öffentlichen Entwicklungshilfe der 17 Mitgliedländer des DAC 31\% ihrer gesamten Netto-Finanzflüsse nach Entwicklungsländern 1981. Für die Schweiz betrug dieser Anteil nur 10,1\% (15).

\section{WIRTSCHAFTS- UND HANDELSPOLITISCHE MASSNAHMEN}

Der auslaufende Rahmenkredit soll durch einen neuen Kredit von 350 Mio Fr. ersetzt werden. Bei der Beratung im Nationalrat wurden die vorgesehenen Massnahmen (Rohstoffe, Handelsförderung, Industrialisierung, Zahlungsbilanz- 
hilfe und Mischkredite) gutgeheissen. Der neue Rahmenkredit bedeutet auch einen wachsenden Anteil - bis zu 15\% - dieser Massnahmen an der gesamten öffentlichen Entwicklungszusammenarbeit.

Ohne Gegenstimme hat der Nationalrat am 23. Juni 1982 einen Rahmenkredit von $350 \mathrm{Mio} F r$. bewilligt, mit dem wirtschafts- und handelspolitische Massnahmen im Rahmen der Entwicklungszusammenarbeit finanziert werden. Der Ständerat folgt in seiner Septembersitzung.

Bei der Behandlung des Kredites im Nationalrat und der zuständigen Kommission kam es zu einer Diskussion über die Mischkredite, einem Teil der vorgesehenen Massnahmen. Ihre entwicklungspolitische Nützlichkeit wurde in Frage gestellt, ebenso die mangelnde Evaluation der bisher gewährten Mischkredite. Es wurde ausgeführt, dass die erhöhten Mischkredite zunehmend ein Dilemma zwischen Handels- und Entwicklungspolitik darstellen. Durch die Gebundenheit der Kredite an die Lieferung schweizerischer Produkte stünden sie im Konflikt zwischen dem Exportinteresse der eigenen Industrie und der entwicklungspolitischen Notwendigkeit im Empfängerland. Es bestünde die Gefahr, dass das Eigeninteresse überwiegt (16). Der Bundesrat wies in der Diskussion auf die geplanten Evaluationen abgeschlossener Mischkredite hin und hält erste Berichte für 1983 möglich (17).

Die Arbeitsgemeinschaft Swissaid/Fastenopfer/Brot für Brüder/Helvetas schlug vor, auch mit dem neuen Rahmenkredit den Anteil der wirtschaftsund handelspolitischen Massnahmen an den gesamten Aufwendungen des Bundes für Entwicklungszusammenarbeit auf $10 \%$ zu belassen und nicht wie vorgesehen - auf $15 \%$ zu steigern. Solange noch keine entwicklungspolitisch positiven Evaluationen der Mischkredite vorliegen, sollten diese nur proportional zum Gesamtkredit erhöht werden. Damit könnte der Rahmenkredit auf 300 Mio Fr. reduziert oder seine Laufzeit um sechs Monate verlängert werden (18). Die Parlamentarier folgten jedoch diesen Ueberlegungen nicht.

Der Rahmenkredit über 350 Mio Fr. hätte ursprünglich am 1. Juli 1982 in Kraft treten und eine Mindestdauer von drei Jahren haben sollen. Die Auszahlungen erstrecken sich auf fünf bis sieben Jahre. Er löst den gleichartigen Rahmenkredit über 200 Mio Fr. ab, der 1978 in Kraft trat und Mitte 1982 voll verpflichtet war.

Die Genehmigung des Rahmenkredits heisst jedoch nicht, dass dieser in den nächsten drei Jahren ausgegeben wird. Er ermöglicht dem Bundesrat nur, die mit den einzelnen Massnahmen vorgesehenen Verpflichtungen einzugehen und konkrete Auszahlungen zu leisten. Die einzelnen Massnahmen erstrecken sich nämlich auf mehrere Jahre, bei ihrer Vorbereitung muss jedoch der Gesamtaufwand geschätzt werden. Und die Höhe des Kredits wird bestimmt durch die im Finanzplan vorgesehenen Gesamtaufwendungen der Entwick- 
lungshilfe, den Anteil der einzelnen Rahmenkredite und die Dauer der Verpflichtungen.

In der laufenden Legislaturperiode sind für wirtschafts- und handelspolitische Massnahmen folgende Ausgaben verpflichtet oder budgetiert worden (in \% des Gesamtbudgets für Entwicklungszusammenarbeit) :

$\begin{array}{lllll}1979 & 1980 & 1981 & 1982 & 1983 \\ 5,2 \% & 9,6 \% & 10,8 \% & 10,4 \% & 13,3 \%\end{array}$

Unter Berücksichtigung der Verpflichtungen aus den anderen laufenden Rahmienkrediten erhöht sich damit der Anteil der wirtschafts- und handelspolitischen Massnahmen jährlich. Der mittelfristige Anteil dieser Massnahmen soll sich, laut Botschaft, auf rund $15 \%$ belaufen. Dem haben die konsultative Kommission für die Handelspolitik und die beratende Kommission für internationale Entwicklungszusammenarbeit zugestimmt. Damit vermindert sich insbesondere der relative Anteil der technischen Zusammenarbeit und der Finanzhilfe (19).

Der auslaufende Kredit von 200 Mio Fr.

Der auslaufende Kredit, der Ende September 1981 zu rund 80\% verpflichtet war, wurde auf die folgenden Massnahmen verteilt :

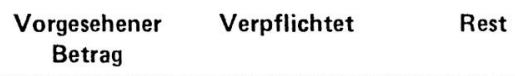

in Millionen Schweizerfranken

\begin{tabular}{lrrr}
\hline & & & \\
Mischkredite & 110 & 102,50 & 7,50 \\
Zahlungsbilanzhilfe & 50 & 33,00 & 17,00 \\
Rohstoffe & 25 & 20,70 & 4,30 \\
Handelsförderung zugunsten & & & \\
der Entwicklungsländer & 10 & 1,20 & 8,80 \\
Industrialisierung & 5 & 2,70 & 2,30 \\
\cline { 2 - 4 } & 200 & 160,10 & 39,90 \\
\hline
\end{tabular}

Quelle : BBL Nr. 12, 1982, s. 726.

In den letzten beiden Jahren wurden die Mittel der betreffenden Rahmenkredite wie folgt verwendet (1981) oder budgetiert (1982) (20) : 


$\begin{array}{lrr} & 1981(\text { Fr. } & 1982(\text { Fr. }) \\ \text { Rohstoffe } & 100.000 & 11.500 .000 \\ \text { Handelsförderung } & 825.067 & 2.000 .000 \\ \text { Industrialisierung } & 694.663 & 2.000 .000 \\ \text { Zahlungsbilanzhilfe } & 18.219 .698 & 9.000 .000 \\ \text { Mischkredite } & 14.582 .542 & 19.100 .000 \\ \text { Durchführung } & & 124.000\end{array}$

Mit Massnahmen im Rohstoff-Bereich werden die Beiträge der Schweiz an internationalen Rohstoffübereinkommen finanziert sowie Projekte, welche die Produktion, den Transport und die Vermarktung von Rohstoffen verbessern sollen. Wichtigste Massnahme wird der schweizerische Beitrag an den Gemeinsamen Rohstoff-Fonds wein, der noch nicht zur Auszahlung gelangte. Die Beiträge zu den Internationalen Uebereinkommen über Naturkautschuk und Zinn werden ebenfalls aus diesem Fonds finanziert.

Im Rahmen der Handelsförderung ist die finanziell wichtigste Massnahme die Beteiligung des Bundes an der Vorbereitung und an der Teilnahme von drei Entwicklungsländern - Aegypten, Senegal und Sri Lanka - an schweizerischen Messen. Weiter wurde ein vom GATT durchgeführter Kurs über Handelspolitik für lateinamerikanische Exportfachleute finanziert, Projekte des ITC (Internationales Handelszentrum UNCTAD/GATT, Genf) und der OS3 (Organisation Schweiz-Dritte Welt, Sonceboz) zum vermehrten Import von Juteprodukten auf dem internationalen und insbesonders dem schweizerischen Markt unterstützt.

Im Bereich der Industrialisierung will man mittels Technologietransfer und schweizerischen Privatinvestitionen die Industrialisierungsbemühungen der Entwicklungsländer stärken. Die Vermittlertätigkeit übernimmt das UNIDOBüro in Zürich. Ihm steht ein spezieller Fonds für die Finanzierung von Vorinvestitionsstudien für Industrieprojekte zur Verfügung. Daraus wurden bisher drei industrielle Investitionsprojekte in Sri Lanka unterstützt (Herstellung von Verpackungen für den Tee-Export, Musikdosenproduktion zum Export, zuckerverarbeitende Fabrik).

Die Zahlungsbilanzhilfe bezweckt, die Importfähigkeit einzelner Entwicklungsländer im Rahmen international koordinierter Massnahmen zu sichern und damit ihre Produktionskapazitäten besser auszulasten. Gleichzeitig hilft sie die Zahlungsbilanzschwierigkeiten zu beheben und den internationalen Finanz- und Handelsverkehr des Entwicklungslandes aufrechtzuerhalten. Sie entspricht auch dem schweizerischen Interesse an einem funktionierenden internationalen Zahlungssystem und einer liberalen Handelspolitik.

Im Rahmen eines Importprogrammkredits der IDA von 74 Mio US-Dollar erhielt Bangla Desh einen nicht rückzahlbaren Beitrag von 15 Mio Fr. (9 Mio US-Dollar).Damit sollen im Rahmen eines nationalen Programms zur Förderung der Nahrungsmittelproduktion Importe von Rohstoffen, Halbfertig- 
produkten und Ersatzteilen getätigt werden (21).

Zu den bis Ende Juni 1981 gewährten neun Mischkrediten kamen bis Ende Juni 1982 weitere vier hinzu : Mischkredite an Zimbabwe (1. und 2. Abkommen), Kenya, Marokko und Honduras. Der Kredit an Aegypten ist aufgebraucht, die anderen Kredite befinden sich in Abwicklung. Ausbezahlt wurden weitere Kredittranchen an Indien, Tunesien, Thailand, Sri Lanka und Senegal (22).

Gegen den Mischkredit an Marokko wurde am 10. August 1981 vom "Schweizerischen Unterstützungskomitee für die Saharaouis" eine Petition eingereicht, welche den Abbruch der Entwicklungszusammenarbeit mit Marokko fordert, solange dieses sich in kriegerischen Auseinandersetzungen befindet. Der Bundesrat wurde aufgefordert, den saharaouischen Flüchtlingen humanitäre Hilfe zukommen zulassen (23).

Auch im Parlament wurde dieser Mischkredit in persönlichen Vorstössen kritisiert, sei es wegen der kriegerischen Auseinandersetzung, sei es wegen der exportorientierten Entwicklungspolitik Marokkos. Der Bundesrat glaubt jedoch, trotz des Krieges habe der Kredit eine positive Wirkung auf die Wirtschaftspolitik Marokkos, die er als entwicklungsfördernd einschätzt (24).

\section{Der neue Rahmenkredit}

Der neue Rahmenkredit, grundsätzlich in die fünf gleichen Bereiche Rohstoffe, Handelsförderung zugunsten der Entwicklungsländer, Industrialisierung, Mischkredite und Zahlungsbilanzhilfe aufgeteilt, sieht folgende Ausgaben vor (25) :

Mischkredite und ähnliche Massnahmen
Zahlungsbilanzhilfe
Rohstoffe
Handelsförderung
Industrialisierung

$$
\begin{array}{r}
240 \text { Mio } \\
70 \text { Mio } \\
20 \text { Mio } \\
10 \text { Mio } \\
10 \text { Mio }
\end{array}
$$

Die einzelnen Massnahmen haben verschiedene Entwicklungsziele zum Ziel : "Mit der Finanzhilfe, die Mischkredite eingeschlossen, tragen wir zum Ausbau der sozialen und wirtschaftlichen Infrastruktur bei. Wirtschafts- und handelspolitische Massnahmen bezwecken, die Entwicklungsländer besser am Welthandel zu beteiligen, damit sie aus inm grösseren Nutzen ziehen können, sowie Störungen im internationalen Handels- und Zahlungsverkehr zu mildern. Schliesslich soll der Einsatz privatwirtschaftlicher Mittel, welche die Entwicklung im Sinne der Zielsetzungen des Entwicklungszusammenarbeitsgesetzes begünstigen, gefördert werden" (26).

Die Empfängerländer dieser Massnahmen sollen bereits gewisse Forischritte im Entwicklungsprozess gemacht haben. Sie richten sich eher an diejenigen ärmeren Länder, die bereits ein etwas höheres Pro-Kopf-Einkommen aufweisen (eine mögliche Ausnahme : Zahlungsbilanzhilfe). Oft lassen sie sich 
nur auf alle Entwicklungsländer oder zumindest auf eine Gruppe von ihnen anwenden (indirekte Wirkungsweise). Die Beziehungen zur schweizerischen Wirtschaft im engeren Sinne, wie auch die allgemeinen aussenwirtschaftspolitischen Interessen des Landes stehen bei diesen Massnahmen stärker im Vordergrund als die humanitären und politischen Motive der Entwicklungszusammenarbeit (27).

\section{REGIONALE ENTWICKLUNGSBANKEN}

Die Interamerikanische Entwicklungsbank (IDB), deren Mitglied die Schweiz seit 1976 ist, gelangte 1981, wie schon in früheren Jahren, auf den schweizerischen Kapitalmarkt : In diesem Jahr nahm sie für 129,9 Mio US-Dollar Kapital in der Schweiz auf :

- 7\% Anleihe 1981-91 von 100 Mio SFr. eines Konsortiums unter der Leitung von drei Schweizer Grossbanken,

- 81/4\% Privatplazierung 1981-86 von 75 Mio SFr. eines schweizerischen Bankenkonsortiums unter der Führung der Schweiz. Bankgesellschaft,

- 81/2\% Anleihe 1981-91 von 80 Mio SFr. eines Konsortiums der drei Grossbanken.

Damit beliefen sich Ende 1981 die ausstehenden Anleihen der IDB auf 3,476 Mia US-Dollar, wovon 0,8 Mia in der Schweiz ausstehen. Der Finanzplatz Schweiz war damit - nach den USA - der wichtigste Kreditgeber der Bank. Im ersten Halbjahr 1982 nahm die IDB weitere Anleihen in der Schweiz auf (28) :

- 7\% Anleihe 1982-92 von 100 Mio SFr.,

- 8\% Privatplazierung 1982-87 von 50 Mio SFr.

An der Jahrestagung der IDB (29.-31. März 1982 in Cartagena/Kolumbien) stand die sechste Kapitalaufstockung im Mittelpunkt der Beratungen. Während die USA eine restriktive Finanzpolitik der Entwicklungsbank befürworteten, drängten die regionalen Mitgliederländer auf eine Ausweitung der Kredite. Meinungsverschiedenheiten bestanden auch beim Prozentsatz des von den Mitgliedern einzuzahlenden Kapitals und bei der Promovierungspolitik, d.h. beim Zugang der einzelnen Länder zu den Krediten. Die Diskussionen werden fortgesetzt.

Die Schweiz trat für eine substantielle Kapitalerhöhung und eine Selbstdisziplin der reicheren, regionalen Mitgliederländer ein. Eine Senkung des Anteils des einzuzahlenden Kapitals könne die Stellung der Bank auf den Kapitalmärkten beeinträchtigen. Die Schweiz plädierte auch für eine bessere Vertretung der nicht-regionalen Mitglieder in der Bankleitung. Sie setzte sich für einen dritten Sitz dieser Länder in der Leitung der Entwicklungsbank ein. An den Spezialfonds der Bank zahlte die Schweiz letztes Jahr einen Beitrag 
von 7,3 Mio Fr. (29).

Die asiatische Entwicklungsbank (ADB), deren Mitglied die Schweiz seit 1967 ist, hat 1981 und im ersten Halbjahr 1982 folgende Anleihen in der

Schweiz aufgenommen :

$-7,75 \%$ - Anleihe von 80 Mio Fr. 1981-91,

- 8\% - Anleihe von 80 Mio Fr. 1981-90 von einem schweiz. Bankenkonsortium unter der Führung der Kreditanstalt,

- 7\% - Anleine von 100 Mio Fr. 1982-92.

An der Jahrestagung der ADB Ende April in Manila wurde die dritte Wiederauffüllung des Asiatischen Entwicklungsfonds (ADF), mit welchem die Bank den ärmsten Ländern weiche Kredite gewährt, beschlossen. Für seine Wiederauffüllung werden 3,2 Mia US-Dollar bereitgestellt. Die Schweiz stellt 10,0 Mio Fr. zur Verfügung. Damit bleibt die Kreditvergabe real im bisherigen Rahmen bestehen. Eine ursprünglich von der Bank erhoffte Erhöhung des Kredits scheiterte nach einjährigen Verhandlungen an der restriktiven Haltung der USA, die jetzt auch prozentual weniger Mittel bereitstellen als bisher (30).

An der Jahrestagung der Afrikanischen Entwicklungsbank (BAD), am 5.-8. Mai 1982 in Lusaka/Zambia, wurde die Oeffnung dieser Entwicklungsbank für nicht-regionale Mitglieder ermöglicht. Die Schweiz hatte bereits 1980 beschlossen, dieser Entwicklungsbank beizutreten. Ausserdem wurde die 3. Wiederauffüllung des Afrikanischen Entwicklungsfonds (FAD) erfolgreich abgeschlossen. Die Auffüllungssumme von 1,07 Mia US-Dollar gestattet dem Fonds die Fortsetzung seiner Tätigkeit, jedoch ohne realen Zuwachs seiner Mittel. Der Beitrag der Schweiz beläuft sich auf 26,9 Mio Fr. Die Schweiz kann ausserdem bei dieser Entwicklungsbank während der nächsten zwei Jahre einen Exekutivdirektor stellen (31).

\section{TECHNISCHE ZUSAMMENARBEIT UND FINANZHILFE}

Auf der Basis des laufenden Rahmenkredits konzentrierte sich 1981 die technische Hilfe im bilateralen Rahmen auf den landwirtschaftlichen Sektor und die Schwerpunktländer der Hilfe. Bei der multilateralen Finanzhilfe waren - neben dem UNDP - die Leistungen an den FAD und die IFAD von grösserer Bedeutung.

1981 wickelte sich die Tätigkeit der DEH - der Bundesstelle, welche fast ausschliesslich die öffentliche technische Zusammenarbeit und Finanzhilfe durchführt - vor dem Hintergrund des Ende 1980 bewilligten Rahmenkredits von 1650 Mio Fr. ab. Bis Ende Juni 1982 wurde dieser Kredit mit 549 Mio 
Fr. für Aktionen beansprucht, von denen sich einige bis ins Jahr 1986 erstrecken werden (32).

Von der DEH - mit ihren 4.155 Mitarbeitern in der Zentrale und im Feld wurden letztes Jahr 435 bilaterale Projekte durchgeführt, davon 50 neue. 406 Projekte lassen sich der technischen Zusammenarbeit zuteilen, 29 der Finanzhilfe (33). Die folgende Tabelle gibt die Auszahlungen der DEH für technische Zusammenarbeit und Finanzhilfe im Jahr 1981 wieder :

\begin{tabular}{|c|c|c|c|}
\hline & $\begin{array}{l}\text { Teshnisshe } \\
\text { Zusammen- } \\
\text { Arbeit }\end{array}$ & Finanzhilfe & Total \\
\hline Bilaterale Tätigkeit & 153,5 & 46,5 & 200,0 \\
\hline Multilaterale Tätigkeit & 37,2 & 54,0 & 91,2 \\
\hline Entwicklungszusammenarbeit & 190,7 & 100,5 & 291,2 \\
\hline
\end{tabular}

Quelle: Geschäftsbericht des Bundesrat 1981, s. 30.

\section{Bilaterale Entwicklungszusammenarbeit}

Die bilaterale (200 Mio) und dabei insbesondere die technische (153,5 Mio) Entwicklungszusammenarbeit stellen seit Jahren die wichtigsten Formen schweizerischer Entwicklungszusammenarbeit dar. Die DEH führt 1981 188 Aktionen selbst durch; 61 Aktionen der technischen Zusammenarbeit wurden an schweizerische Institutionen und Firmen in Regie vergeben und für 102 Projekte wurden namentlich an private Hilfswerke und öffentliche Körperschaften und Institutionen Beiträge bezahlt. Schliesslich wurden an 84 technische oder Finanzhilfe-Projekte internationaler Organisationen Beiträge bezahlt. Mit der Privatwirtschaft besteht eine Zusammenarbeit hauptsächlich in Form von Materialeinkäufen und von Aufträgen an Ingenieurbüros und private Beraterfirmen (1982 : 27 Mio Fr.) (34).

Die Aufteilung der bilateralen technischen Zusammenarbeit und Finanzhilfe nach Kontinenten hat sich 1981 gegenüber dem Vorjahr leicht verändert, indem Afrika - auf Kosten Asiens - 1981 einen um 5,5\% höheren Anteil an den Auszahlungen aufweist. Die Auszahlungen nach Asien nahmen auch umfangmässig ab (vgl. auch die Statistiken). 


\section{AKTIONEN FUER DIE BILATERALE TECHNISCHE ZUSAMMENARBEIT UND FINANZHILFE 1981 : AUFTEILUNG NACH KONTINENTEN}

(Nettoauszahlungen in Mio Fr.)

\begin{tabular}{lcr} 
Region & Mio Fr. & $\%$ \\
\hline Afrika & 90,4 & 45,2 \\
Lateinamerika & 28,2 & 14,1 \\
Asion & 57,7 & 28,9 \\
Europa & 1,8 & 0,9 \\
Nicht klassiert & 21,8 & 10,9 \\
\cline { 2 - 3 } TOTAL & 200,0 & 100,0 \\
\hline
\end{tabular}

Quelle : Jahresbericht des DEH 1981.

In die 17 "Schwerpunktländer" der schweizerischen Entwicklungszusammenarbeit flossen dabei 1981 82,3\% aller Auszahlungen. Nach verschiedenen Einkommensgruppen aufgeteilt, ergibt sich für die Auszahlungen an bilateraler Entwicklungszusammenarbeit folgendes Bild:

Bilaterale technische Zusammenarbeit und Finanzhilfe :

\section{AUSZAHLUNGEN GEGLIEDERT NACH EINKOMMENSTAND}

\section{DEM EMPFAENGERLAENDER}

(in Mio Fr., 1981)

Ländergruppen

Technische Finanzhilfe TOTAL \%
Zusammen-
arbeit

Am wenigsten entwickelte Länder 54,9 31,1

86,0 43,0

Andere Länder mit tiefem Einkommenstand (BSP pro Kopf unter $\$ 500$ ) 33,6 7,8 41,4 20,7

Länder mit mittlerem Einkommen (BSP pro Kopf über \$500) 22,9 2,4 25,3 12,6

Regionale Projekte und nicht klassiert

TOTAL

\begin{tabular}{rrrr}
42,1 & 5,2 & 47,3 & 23,7 \\
\hline 153,5 & 46,5 & 200,0 & 100,0
\end{tabular}

Quelle : Jahresbericht der DEH 1981, Tabelle 6. Die aufgeführten Ländergruppierungen entsprechen den von der OECD verwendeten Definitionen und beziehen sich auf das BSP von 1979. 
Bei der sektoriellen Aufteilung der Ausgaben ist eine Steigerung des wichtigsten Sektors, der Landwirtschaft, auf einen Anteil von 35\% festzustellen. Die Anteile der übrigen Sektoren veränderten sich wenig. Hingegen büssten die multisektoriellen Projekte anteilsmässig an Bedeutung ein ( $\mathrm{vgl}$. auch die Statistiken).

\section{Assoziierte Hilfe und Ko-Finanzierung}

Assoziierte Hilfe und Ko-Finanzierung sind weitere Teile der bilateralen Entwicklungszusammenarbeit. Assoziierte Hilfe will heissen : “Finanzierung eines Projekts, das von einer internationalen Organisation durchgeführt und verwaltet, aber von der DEH ausgewählt und kontrolliert wird" (35). Mitfinanzierung bedeutet die gleichzeitige finanzielle Beteiligung mehrerer (biund/oder multilateraler) Geldgeber, die sich ausserhalb des Empfängerlandes befinden, an einem Projekt, gemäss eines im Einvernehmen mit dem Empfänger geschlossenen Vertrages.

Assoziierte Hilfe leistet die Schweiz in Zusammenarbeit mit mehreren UNSonderorganisationen und Institutionen, z.B. mit der FAO, der UNICEF und dem BIT. Ende 1981 befanden sich über 55 solche gemeinsamen Projekte in Abwicklung, davon 23 unter der Leitung der FAO, 10 unter der Leitung des BIT und 8 von UNICEF geleitete. Die Auszahlungen betrugen 1981 für assoziierte Hilfe und Ko-Finanzierungen 42,6 Mio Fr., wie die folgende Tabelle zeigt. Hauptempfänger waren UN-Institutionen (FAO, BIT, UNICEF), internationale Forschungsinstitute und die Institutionen für Entwicklungsfinanzierung (Regionale Entwicklungsbanken und die Weltbanktochter (DA).

Mitfinanzierend beteiligt sich die DEH an der Finanzierung einiger Projekte regionaler Entwicklungsbanken und der IDA. Die entsprechenden Projekte wählt die DEH selbst aus. Die Finanzierung erfolgt grundsätzlich ungebunden. Die Realisierung erfolgt unter Aufsicht der Entwicklungsbank durch das Empfängerland; schweizerische Unternehmen können sich an den Projektausschreibungen beteiligen. 


\section{ASSOZIIERTE HILFE UND KO-FINANZIERUNG \\ MIT INTERNATIONALEN ORGANISATIONEN}

(Nettoauszahlungen in Mio Fr., 1981)

$\begin{array}{ll}\text { Assoziierte } & \begin{array}{l}\text { Ko-finanzie- } \\ \text { rungen }\end{array}\end{array}$

\begin{tabular}{lccc}
\hline Institutionen der Vereinten Nationen (UNO) & 18,0 & - & 18,0 \\
$\begin{array}{l}\text { Organisation der UNO für Ernährung } \\
\text { und Landwirtschaft (FAO) }\end{array}$ & 6,6 & - & 6,6 \\
Kinderhilfswerk der UNO (UNICEF) & 4,0 & - & 4,0 \\
$\begin{array}{l}\text { Internationales Arbeitsamt (ILO) } \\
\text { Konferenz der UNO für Handel }\end{array}$ & 3,3 & - & 3,3 \\
und Entwicklung (UNCTAD) & 0,8 & - & 0,8 \\
Andere & 3,3 & - & 3,3 \\
Institutionen für Entwicklungsfinanzierung & - & 8,6 & 8,6 \\
Internationale Entwicklungsagentur (IDA) & - & 5,0 & 5,0 \\
Afrikanische Entwicklungsbank (BAD) & - & 0,5 & 0,5 \\
Asiatische Etwicklungsbank (ADB) & - & 3,1 & 3,1 \\
Internationale Forschungsinstitute & 14,9 & - & 14,9 \\
Konsultativgruppe für internationale & 12,6 & & 12,6 \\
landwirtschaftliche Forschung (CGIAR) & 2,3 & - & 2,3 \\
Andere & 1,1 & - & 1,1 \\
Andere internationale Organisationen & 34,0 & - & 42,6 \\
\hline TOTAL & & 8,6 & \\
\hline
\end{tabular}

Quelle : Geschäftsbericht des Bundesrat 1981, s. 39.

Nachdem 1980 die interamerikanische und die asiatische Entwicklungsbank nichtrückzahlbare Beiträge für Projekte tẹchnischer Hilfe und kleinere Finanzhilfeprojekte erhalten hatten, wurde im August 1981 der asiatischen Entwicklungsbank eine gleichartige Hilfe gewährt (36).

\section{Multilaterale Entwicklungszusammenarbeit}

Die multilaterale technische Entwicklungszusammenarbeit und die Finanzhilfe beliefen sich 1981 auf 91,2 Mio Fr., wovon 37,2 Mio Fr. für die technische Zusammenarbeit und 54 Mio Fr. für die Finanzhilfe aufgewendet wurden. Hauptempfänger war das UNDP, mit 30,2 Mio Fr., neben dem afrikanischen (26,9 Mio Fr.) und dem asiatischen Entwicklungsfonds (10 Mio Fr.). 
Dem UNDP standen für das Jahr 1981 nur ca. 550 Mio Dollar zur Verfügung. Die Schweiz finanzierte 1981 2,1\% aller Beiträge (37). Die DEH koordiniert und verwaltet die Kredite für die multilaterale Finanzhilfe, welche gemeinsam von DEH und BAWI getragen werden. Neben den Beiträgen an die verschiedenen regionalen Entwicklungsfonds war die Wiederaufstockung der Mittel des Internationalen Fonds für landwirtschaftliche Entwicklung (IFAD) von Bedeutung. In den langwierigen Verhandlungen, bei denen es vor allem um die Verteilung der Geber-Anteile zwischen OPEC- und OECD-Länder ging, setzte sich die Schweiz dafür ein, dass der Fonds seine Tätigkeit mindestens im bisherigen Rahmen fortsetzt. Seit 1981 ist die Schweiz auch durch einen stellvertretenden Direktor im IFAD-Verwaltungsrat vertreten. Beim Fonds verfügen die OECD- und OPEC-Länder sowie die übrigen Entwicklungsländer über gleichviel Stimmen. Ziel des Fonds ist es, die einheimische Nahrungsmittelproduktion zu fördern und seine Tätigkeit auf die Befriedigung der Grundbedürfnisse auszurichten (38).

Der Bundesrat hat Ende Juni 1982 einen schweizerischen Beitrag von 28,44 Mio Fr. (15,5 Mio Dollar) an die erste Wiederauffüllung des IFAD genehmigt. Dies entspricht einem Anteil von 2,5\% am gesamten Beitrag der OECDLänder. An seiner 5. Session (19.-22. Januar 1982 in Rom) beschloss der Gouverneursrat der IFAD ausserdem, Rom zum definitiven Sitz des Fonds zu bestimmen. Die beschlossene Wiederaufstockung - die OECD-Länder bringen 58\%, die OPEC-Länder 42\% -- von 1,100 Mio Dollar, muss verläufig ohne die Beiträge der USA durchgeführt werden. Diese haben zwar der Aufstockung zugestimmt, die erforderlichen Mittel jedoch nicht budgetiert (39).

Multilaterale technische Zusammenarbeit und Finanzhilfe :

ALLGEMEINE BEITRAEGE AN INTERNATIONALE ORGANISATIONEN

(Nettoauszahlungen in Mio Fr., 1981)

Technische

Finanzhilfe

TOTAL

Zusammen-

arbeit

Organisationen der Vereinten Nationen (UNO)

37,2

6,8

44,0

Entwicklungsprogramm der UNO (UNPD)

30,2

30,2

Fonds für die am wenigsten

entwickelten Länder

Fonds für Wissenschaft und Technologie

Ausrüstungsfonds der UNO (UNCDF)

Bevölkerungsfonds der UNO (UNFPA)

Spezialprogramm zur Erforschung von

Tropenkrankeiten der Weltgesundheits-

organisation (WHO)

Andere Spezialfonds der UNO

4,0

0,8

-

-

1,7

0,5
4,0

0,8

3,8

3,0

1,7

0,5 
Regionale Entwicklungskanken

und ihre Spezialfonds

$\begin{array}{lll}- & 47,2 & 47,2\end{array}$

Afrikanischer Entwicklungsfonds (FAD) - $\quad 26,9 \quad 26,9$

Asiatische Entwicklungsbank (ADB) - $\quad 2,3 \quad 2,3$

$\begin{array}{lll}\text { Asiatischer Entwicklungsfonds (ADF) } & \text { - } & 10,0\end{array}$

$\begin{array}{llll}\text { Interamerikanische Entwicklungsbank (IDB) - } & 0,7 & 0,7\end{array}$

Fonds für Spezialoperationen der IDB

$7,3 \quad 7,3$

TOTAL

37,2

54,0

91,2

Quelle : Jahresbericht der DEH 1981, Tabelle 16.

\section{HUMANITAERE HILFE}

In der Wintersession 1981, am 3. Dezember, gewährten die eidgenössischen Räte einen neuen Rahmenkredit von 360 Mio Fr. für internationale humanitäre Hilfe, nachdem der Bundesrat eine entsprechende Botschaft veröffentlicht hatte. Dieser neue Rahmenkredit wurde notwendig, da der letzte - in der Höhe von 270 Mio Fr. - Ende März 1982 auslief. Der jetzige Rahmenkredit hat eine Mindestdauer von drei Jahren. Es ist vorgesehen, diesen Kredit wie folgt auf die verschiedenen Bereiche der humanitären Hilfe aufzuteilen (40) :

Mio Fr.

$\begin{array}{lr}\text { Beiträge an internationale Organisationen und } & 131 \\ \text { international tätige Hilfswerke } & 31 \\ \text { Schweizerisches Katastrophenhilfekorps } & 112 \\ \text { Nahrungsmittelhilfe mit schweizerischen Milchprodukten } & 51 \\ \text { Getreidehilfe } & \\ \text { Reserve für aussergewöhnliche Katastrophenfälle, } & 35 \\ \text { inbegriffen Verpflichtungen im Rahmen der inter- } \\ \text { nationalen Nahrungsmittel-Nothilfe } \\ \text { TOTAL } & 360\end{array}$

Ausserdem stimmte das Parlament im Dezember 1981 der Erhöhung des regelmässigen Beitrags der Schweiz an das IKRK auf 20 Mio Fr. jährlich zu (41).

Insgesamt erfolgten im Bereich der humanitäre Hilfe 1981 folgende Zahlungen : 
für Hilfeleistungen bei Katastrophen

im Ausland

Ordentliche und ausserordentliche Beiträge

an internationale Organisationen und

international tätige Hilfswerke (inkl.

Erneuerung der Nothilfeausrüstung)

Nahrungsmittelhilfe

namentlich schweizerische Milchprodukte und

Getreide (inkl. Geldbeiträge für den Ankauf und Transport von Nahrungsmitteln)

TOTAL

Quelle : Jahresbericht der DEH 1981, Tabelle 17. Dieser Betrag schliesst 2,5 Mio Fr. für Aktionen ein, die nicht in Entwicklungsländern durchgeführt wurden, z.B. Polen.

\section{Internationale Nahrungsmittelhilfe}

Im Bereich der internationalen Nahrungsmittelhilfe leistete die Schweiz einen Beitrag von 46,2 Mio Fr. Dies ist die Hälfte der im letzten Jahr insgesamt für die humanitäre Hilfe geleisteten Zahlungen. Neben Geldspenden wurden dafür 3.410 Tonnen Schweizer Milchprodukte, 21.151 Tonnen Getreideprodukte sowie andere Nahrungsmittel in kleinen Mengen gespendet. Die Verteilung erfolgte über bilaterale und multilaterale Kanäle. Etwa die Hälfte der Lieferungen gingen an die Not- und Flüchtlingshilfe (42).

In einer Studie des Sozialethischen Institute des Schweiz. Evangelischen Kirchenbundes und vom Informationsdienst 3. Welt wird die Nahrungsmittelhilfe zwar als notwendig, aber problematisch eingeschätzt (43). Nach Ansicht der Autoren sollte sich die Nahrungsmittelhilfe beschleunigt auf Nothilfe in Katastrophensituationen beschränken. Bei der Milchpulverhilfe erfolge eine ungenügende Evaluation und vermischen sich schweizerische Wirtschaftsinteressen und Erfordernisse in den Hungergebieten. Dies ist umso mehr bei der schweizerischen Getreidehilfe der Fall. Wenig Verständniss ruft das Versuchsprogramm mit schweizerischen Weissfischkonserven hervor (44).

\section{Operationelle humanitäre Hilfe}

Das Freiwilligenkorps für Katastrophenhilfe im Ausland, welches den operationellen Bereich der humanitären Hilfe ausmacht, musste nur wenig in Aktion treten. Grosse Naturkatastrophen blieben aus. Aktionen zugunsten 
von Flüchtlingen wurden in Thailand, auf den Philippinen, in Pakistan, Kamerun und Somalia durchgeführt.

Die mehrjährige Wiederaufbauaktion in Guatemala nach dem Erdbeben vom Februar 1976 konnte ebenso abgeschlossen werden wie jene zugunsten von El Asnam (Algerien); weitere Einsätze zur Verbesserung von Infrastrukturanlagen erfolgten in Ghana, Zimbabwe und Aequatorial-Guinea.

Die gesamten Aufwendungen der Flüchtlingshilfe betrugen 1981 22,65 Mio Fr., wovon 12,79 Mio Fr. an multilateraler Hilfe vergeben wurden. Von der bilateralen Hilfe gingen 5,84 Mio Fr. nach Afrika (Somalia) und 3,68 Mio Fr. nach Asien (Pakistan, Thailand) (45). Im Juni 1982 bewilligte der Bundesrat einen Zusatzkredit von 5 Mio Fr. für die Nothilfe an die Zivilbevölkerung im umkämpften Libanon (46).

Auf Ende 1981 ist der erste Delegierte des Bundesrates für Katastrophenhilfe im Ausland, Arthur Bill, in den Ruhestand getreten. Zu seinem Nachfolger ernannte der Bundesrat Eduard Blaser (47).

\section{STIPENDIEN}

Mit dem Rahmenkredit von 9,2 Mio Fr. vom 19. Dezember 1980 stehen dem Bundesamt für Kulturpflege des EDI finanzielle Mittel zur Verfügung, um Stipendien an ausländische Studierende in der Schweiz zu vergeben. Grösstenteils gehen diese an Studierende aus Entwicklungsländern. 1981 wurden dafür 3,3 Mio Fr. ausgegeben, einschliesslich eines von der DEH geleisteten Beitrags von $920.000 \mathrm{Fr}$. aus ihrem Stipendienkredit. Für 1982 sind 3,8 Mio Fr. vorgesehen; die DEH leistet ebenfalls wieder einen Beitrag. 1981 wurden vom EDI rund 200 Stipendien vergehen (48).

Im Rahmen der bilateralen technischen Zusammenarbeit und assoziierten Hilfe wurden 1981282 Stipendiaten aus Entwicklungsländern in der Schweiz ausgebildet. Die DEH wendete dafür rund 2,7 Mio Fr. auf. Die DEH-Stipendien werden für Ergänzungsausbildungen für Personen mit Berufserfahrung vergeben.

1981 erhielt der grösste Teil dieser Stipendiaten eine Ausbildung im Dientsleistungsbereich (Hotellerie, Post/Telekommunikation, Gesundheit) oder im technisch/industriellen Bereich. Die meisten Stipendiaten (67\%) kamen aus afrikanischen Ländern, 20\% aus asiatischen. Der Anteil der Frauen betrug 11\%. 1980-81 wurde eine Erhebung über die jetzige berufliche Situation der ehemaligen DEH-Stipendiaten durchgeführt. Die grosse Mehrheit arbeitet demnach in ihrem Heimatland in Bereichen, in denen sie in der Schweiz zusätzlich ausgebildet wurde (49). 


\section{AUFWENDUNGEN VON KANTONEN UND GEMEINDEN}

1981 finanzierten Kantone und Gemeinden mit 6,1 Mio Fr. (oder zu 1,35\%) die öffentliche Entwicklungszusammenarbeit; ihre Beiträge wurden allerdings für Leistungen privater Organisationen verwendet. Der Gesamtbeitrag der Kantone machte dabei 2,78 Mio Fr. aus. Die grössten Beiträge kamen aus den Kantonen Genf (898.000 Fr.), Basel-Stadt (507.000 Fr.) und Zürich (255.000 Fr.) Einzelne Gemeinden leisteten einen Gesamtbeitrag von 3,34 Mio Fr. Sieben Gemeinden lieferten Beiträge ab, die über $100.000 \mathrm{Fr}$. liegen: Genf, Zürich, Bern, Küsnacht $(\mathrm{ZH})$, Zollikon $(\mathrm{ZH})$, Lancy $(\mathrm{GE})$ und Kloten (ZH) (50).

Einzelne Kantone (Basel-Stadt, Jura, Waadt) und eine Gemeinde (Münsingen/ Bern) führen selber Aktionen in Entwicklungsländern durch. Ihre Aufwendungen, inkl. den dafür zur Verfügung stehenden Bundesmitteln, beliefen sich 1981 auf 0,88 Mio Fr. (51).

In Genf wurde 1980 eine kantonale Initiative "Initiative 0,7" lanciert, welche einen kantonalen Entwicklungshilfe-Fonds fordert. Dieser würde aus kantonalen Geldern gespiesen, welche sich auf 0,7\% des kantonalen Einkommens belaufen. Diese Prozentzahl soll schrittweise während der nächsten zehn Jahre erreicht werden.

Am 21. Januar 1982 unterstützte der Grosse Rat die Initiative und die Abstimmung findet am 26. September statt. Die Mittel des Fonds sollen der technischen Zusammenarbeit zugute kommen, welche die eigenen Anstrengungen der Entwicklungsländer und der am meisten benachteiligten Gruppen und Regionen fördert. Projekte über $250.000 \mathrm{Fr}$. müssen vom Grossen Rat genehmigt werden und unterstehen dem Referendumsrecht. Bei einer kantonalen oder schweizerischen Arbeitslosenzahl von mindestens $0,5 \%$ oder besonderen wirtschaftlichen Schwierigkeiten einer schweizerischen Region kann die Hilfe auf $0,6 \%$ gesenkt werden (52).

Im jurassischen Parlament wurde in einer Motion verlangt, 0,1\% des laufenden Budgets für die Entwicklungszusammenarbeit zu verwenden. Dies, solange die Schweiz nicht 0,7\% ihres BSP für die öffentliche Entwicklungszusammenarbeit verwendet (53).

\section{PRIVATE ENTWICKLUNGSZUSAMMENARBEIT}

Die private Entwicklungszusammenarbeit ist eigentlich nicht zu den Leistungen des Bundes zu zählen. Sie ist jedoch mit diesen vielfach verbunden. So werden den privaten Hilfswerken ergänzend zu ihren eigenen Mitteln vom Bund Projekte in Regie überlassen oder Projektbeiträge bezahlt. Auch in der 
entwicklungspolitischen Diskussion stellen die Vertreter der privaten Hilfswerke einen wichtigen Faktor dar.

Die Leistungen der privaten Hilfswerke und Entwicklungsorganisationen beschränken sich nicht nur auf die technische Zusammenarbeit und humanitäre Hilfe. Zahlreich sind ihre Aktivitäten in der Schweiz selbst. Einzelne Organisationen führen gar keine Projekte in Entwicklungsländern durch, sie legen ihre Hauptaufgabe auf die Information der Bevölkerung über Entwicklungsfragen.

1981 konnten die privaten Hilfswerke - religiösen oder neutralen Charakters - 105,6 Mio Fr. an Spendengeldern für ihre Tätigkeit mobilisieren. Während die Höhe der öffentlichen Entwicklungshilfe der Schweiz im Vergleich der OECD-Staaten unterdurchschnittlich ist, rangiert die Schweiz mit ihren Beiträgen an die Hilfswerke an der Spitze, zusammen mit Norwegen. In diesen beiden Ländern machen die Leistungen der privaten Hilfswerke 1981 0,06\% des BSP aus (54).

Um beim wachsenden Volumen der Entwicklungszusammenarbeit und dem fortdauernden Personalstop beim Bund die Qualität und den Anteil der bilateralen Projekte zu gewährleisten, wurde 1981 die Gründung einer "Neuen Organisation" ins Auge gefasst. Sie stand rasch im Zentrum der entwicklungspolitischen Diskussion, insbesondere von Seiten bürgerlicher Parlamentarier und von Ingenieurbüros.

Nachdem sich die beratende Kommission für internationale Entwicklungszusammenarbeit und die ständerätliche und nationalrätliche Kommission für auswärtige Angelegenheiten mit diesem Projekt befasst hatten, stimmte der Bundesrat am 21. Dezember 1981 dem Abschluss eines Kooperationsvertrags zwischen Bund und der Intercooperation zu. Der Verein wurde Anfang 1982 von sieben Hilfswerken (Caritas, Helvetas, HEKS, SAH, Swissaid und Swisscontact) gegründet. Der Bund hatte schliesslich aus juristischen Gründen auf eine Mitgliedschaft verzichtet. Die enge Zusammenarbeit zwischen Bund und "Intercooperation" wird vertraglich geregelt.

In der diesbezüglichen Presse-Dokumentation heisst es : "Bund und Hilfswerke gewähren dem Verein gemeinsam ein Darlehen von je Fr. 500.000.(zusammen Fr. 1.000.000.-); der Bund leistet zusätzlich einen nicht rückzahlbaren Beitrag für die Vorbereitungsphase des Vereins. Das vom Bund dem Verein übertragene Projektvolumen wird in den kommenden Jahren in der Grössenordnung von rund 13-15 Mio Franken liegen. Die Geschäftsstelle des Vereins wird zu Beginn 10-12 Personen umfassen, eine bedeutende höhere Anzahl von Feldmitarbeitern wird in Projekten in Entwicklungsländern eingesetzt sein.

Die Tätigkeit des Vereins wird vor allem bei Landwirtschaftsprojekten (vor allem Viehzuchtprogrammen und Nahrungsmittelproduktion), Forstwirtschaftsprojekten (dörfliche Aufforstungen und Erosionsbekämpfung) und ländlichen Basisentwicklungsprojekten auf verschiedenen Gebieten liegen. 
Solche Aktionen sollen möglichst mit verschiedenen fachlichen Komponenten realisiert und in enger Zusammenarbeit mit der ländlichen Bevölkerung und lokalen Organisationen durchgeführt werden. Sie sind normalerweise nicht allzu kostenaufwendig, aber sehr arbeitsintensiv und erfordern eine sorgfältige Vobereitung und Kontrolle" (55). 